\title{
Patch-Based Model for the Classification of Soybean Leaf Diseases
}

\author{
Gustavo Vigilato G. S. \\ Universidade Tecnológica Federal do Paraná \\ Pato Branco, Brazil \\ gustavo.vigilato@gmail.com
}

\author{
Pablo G. Cavalcanti \\ Universidade Tecnológica Federal do Paraná \\ Pato Branco, Brazil \\ pgcavalcanti@utfpr.edu.br
}

\begin{abstract}
The disease detection is vital to increase the productivity and quality of soybean cultivation and this detection is usually carried out in a laboratory, which is time consuming and costly. To overcome these issues, there is a growing demand for technologies that aim at a faster detection and classification of diseases. In this context, this work proposes the extraction of several patches from a leaf image and combining a convolutional neural network with a support vector machine, we present a complete model for the classification of soybean leaf diseases. In this approach, an image dataset with evidence of diseases commonly observed in soybean crops was analyzed and our experiments achieved precisions greater than $90 \%$.

Index Terms-Soybean leaf diseases, Classification, Convolutional Neural Network.
\end{abstract}

\section{INTRODUCTION}

Soybean is the main agricultural export product of Brazil, filling more than 35 million hectares in 2021 [1]. In the last 20 years, the annual growth of soybean production in Brazil was 3.5 million tons, which represents an increase of $13.4 \%$ each year [2]. Much of this growth is due to the increase and improvement of technology used in the field, such as the use of intelligent irrigation systems and soil quality control.

However, there are several diseases that affect and make it difficult to obtain high levels of productivity in soybeans [3]. Moreover, considering the increase of number of farms, the number of diseases to which soybeans are exposed also increases significantly, and may cause great economic losses. Consequently, the continuous monitoring of the crop is a fundamental strategy for disease management and should be considered among all farmers [3]. However, the process of identifying a disease usually requires an expert and can be very time-consuming, thence also costly [4].

In recent years, precision agriculture has been more widely used, applying computer resources for a better control of the crop productivity and reducing the need for specialized help [5]. And computer vision is frequently applied as part of the precision agriculture, improving many tasks from carrying out weed detection using aerial photos to identify diseases in fruits and plants.

Therefore, several works have been presented over the last few years towards the classification of diseases that affect different types of crops through the analysis of leaves. Fuentes et al. [6] proposed the used of neural networks like Fast-RCNN for the detection of diseases and pests in tomato crops. Hassan et al. [7] analyzed different plants through convolutional neural networks such as Inceptionv3 and MobileNetV2.

Considering the analysis of soybean diseases in Brazil, the present approaches used only classic methodologies (not based on Deep Learning). Pires et al. [4] used Bag of Visual Words and Support Vector Machine (SVM) to achieve 98\% in four types of diseases. Barbedo et al. [8] converted the original RGB images into the HSV, $\mathrm{L}^{*} \mathrm{a} * \mathrm{~b} *$ and CMYK color spaces and using a modified pairwise voting system based on intensity histograms, achieved 58\% of precision for 9 classes of diseases.

A more recent approach proposed by Karlekar et al. [9] was the first to introduce the use of a deep learning convolution neural network $(\mathrm{CNN})$ for soybean leaf diseases classification after a segmentation step to remove the complex background and result in $97 \%$ of precision. However, their experiments created synthetic images using a huge data augmentation for both training and testing, thus creating a data leak and making it difficult to analyze their results in real situations.

To the best of our knowledge, this paper is the first to present an approach based on convolutional neural networks (as Karlekar et al. [9]) combined with SVM (as Pires et al. [4]) to identify the most frequent soybean leaf diseases. Moreover, our experiments demonstrate that this robust methodology is able to achieved high precision with much smaller datasets than previous works [4] [6] [7] [9].

\section{Materials And Methods}

The image dataset was originally collected by EMBRAPA (the Brazilian Agricultural Research Corporation) and presents 5 types of leaf diseases and also a class with healthy leaves. The number of images per class is presented in Table I and some image samples can be seen in Figure 1. By the time we had access to this dataset, there were also images with Copper Phytotoxicity, Mirothecium Leaf Spot, Charcoal Rot and Rhizoctonia Aerial Blight disease, however they were not considered in this work due to low number of images (less than 10).

\section{A. Data preprocessing}

Before we could process the images, the dataset was splitted into training, validation and testing sets. The number of images per class after the splitting can be seen in Table II and was 

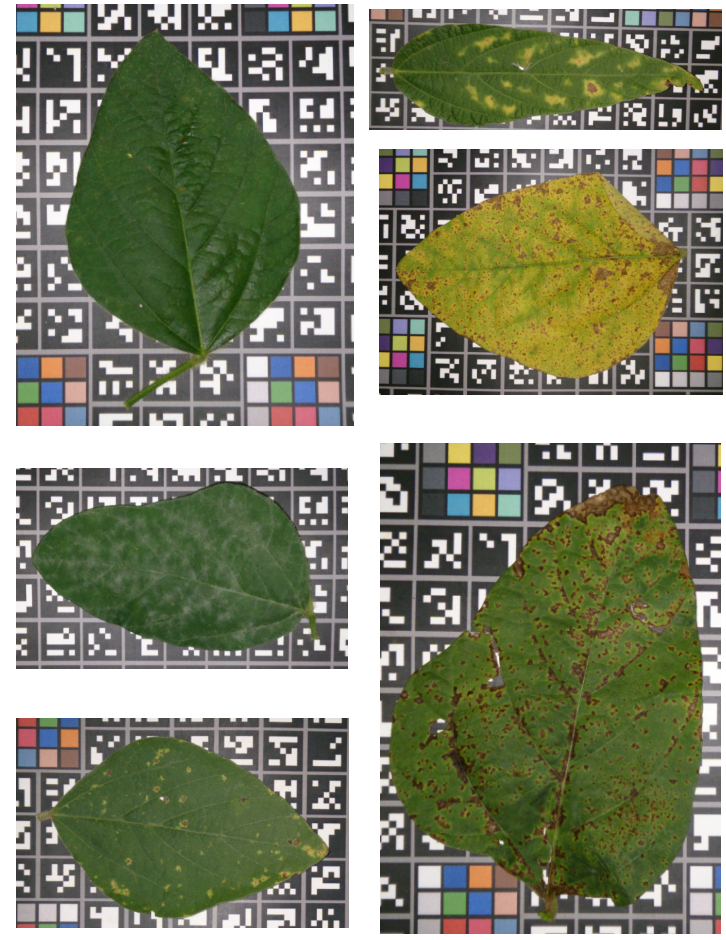

Fig. 1. Example of soybean leaves from the image dataset.

TABLE I

IMAGE DATASET

\begin{tabular}{|c|c|}
\hline Diseases & Number of images \\
\hline Mosaic Virus & 22 \\
\hline Downy Mildew & 35 \\
\hline Powdery Mildew & 78 \\
\hline Rust & 65 \\
\hline Bacterial Blight & 50 \\
\hline Healthy & 8 \\
\hline
\end{tabular}

done manually, so we could guarantee that different levels of disease degradation are present in the training set. However, it is possible to observe that most of the images are separated for training in order to provide a proper estimation of the effectiveness of the proposed architecture.

TABLE II

DATASET SPLITTING

\begin{tabular}{|l|c|c|c|}
\hline Class & Training & Validation & Testing \\
\hline Mosaic Virus & 8 & 4 & 10 \\
\hline Bacterial Blight & 10 & 5 & 35 \\
\hline Rust & 14 & 7 & 47 \\
\hline Downy Mildew & 12 & 6 & 17 \\
\hline Powdery Mildew & 14 & 7 & 57 \\
\hline Healthy & 5 & 3 & 0 \\
\hline
\end{tabular}

However, the low number of samples for training does not allow the training of a deep neural network using the entire images. So, we decided to extract patches from all images, as proposed in [4], and its main objective is to obtain local data (texture information) from each area of the leaf, while also increases the number of samples for training. Nevertheless, this extraction is processed only after the segmentation step. Figure 2 illustrates these steps as well the following steps, and each one of them is described next.

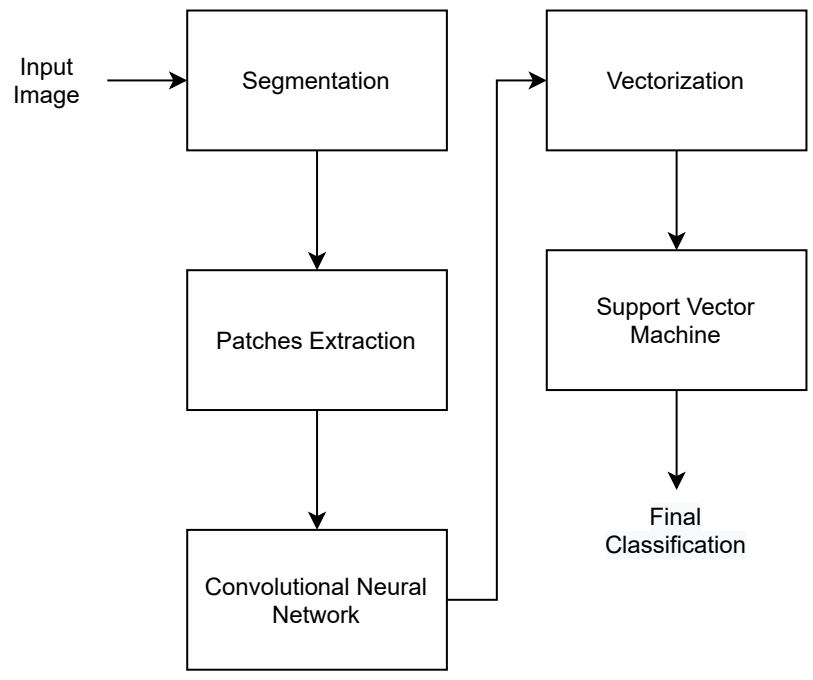

Fig. 2. Proposed architecture diagram.

\section{B. Segmentation}

After careful analysis of several images in the RGB and other color spaces, we observed that the saturation (S) channel from the HSV color model presents a good separation between the leaves pixels and the background pixels. Thus, all pixels with $\mathrm{S}$ higher than 0.7 were considered as part of the leaves and mathematical morphology was used to eliminate possible smaller remaining segments.

An example of the result of the segmentation step is presented in Fig. 3.

\section{Patches extraction}

From each image and its correspondent segmentation mask, patches of $224 \times 224$ pixels were extracted. The stride (number of pixels between each patch) was set to 112 pixels for the training set and 224 for the validation set.

Also, a data cleaning step was necessary, since many patches were extracted near the border of the leaf and may contain several pixels from the background. So, every patch with more than $50 \%$ of background pixels was eliminated. Also, a manual inspection eliminated all patches that were clearly health areas of the leaf. This step aims to help the disease classification, otherwise healthy areas would be part of the set of all diseases and the training process would be very noisy and ineffective.

The preprocessed image dataset contains 5411 patches for training e 892 for validation. The number of patches per class can be seen in Table III.

\section{Patches classification}

We propose the use of InceptionNetV3 [10] for the classification of each extracted patch, since this neural network is one of the most robust deep learning techniques and is part of the state-of-the-art for many classification tasks. But, since its 


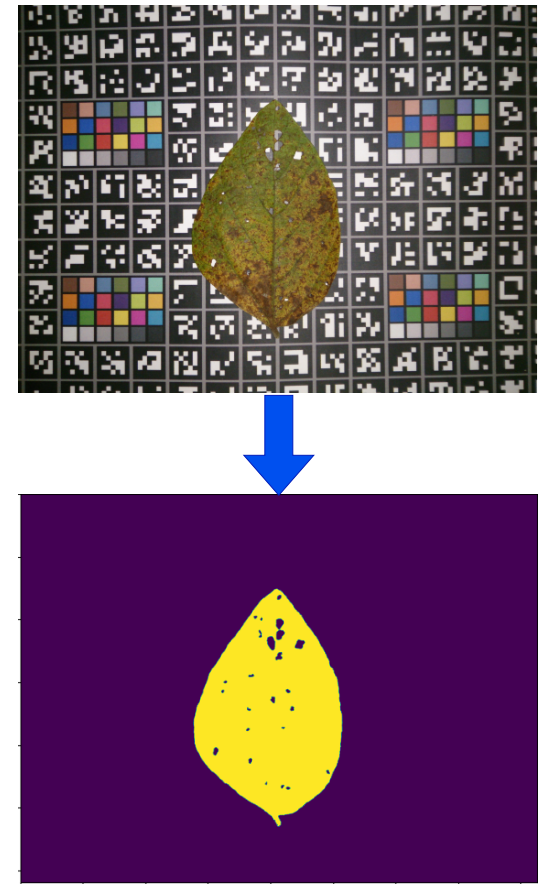

Fig. 3. Illustration of segmented image.

TABLE III

NUMBER OF PATCH IMAGES

\begin{tabular}{|l|c|c|}
\hline Class & Training & Validation \\
\hline Soybean Mosaic Virus & 591 & 80 \\
\hline Bacterial Blight & 1274 & 180 \\
\hline Rust & 913 & 246 \\
\hline Downy Mildew & 907 & 148 \\
\hline Powdery Mildew & 1023 & 163 \\
\hline Healthy & 703 & 75 \\
\hline
\end{tabular}

native input is $299 \times 299$ pixels, all patches were rescaled to this size. Furthermore, we performed data-augmentation to the training set using random zooms, flips and rotations, ensuring a sixfold increase for each class. It is important to observe, though, that the validation data remained intact, to guarantee the technique feasibility in real data.

Based on the concept of transfer learning, the original final fully connected layer with 1000 nodes was replaced with a layer with 6 nodes, representing each of the classes in our dataset. All weights obtained with the ImageNet database for this convolutional neural network were frozen, and only the new final layer is trained for 25 epochs.

As can be seen in Fig. 4, the accuracies for the training and validation sets achieved more than $90 \%$ after only 5 epochs. We continued the training for more 20 epochs so the loss could decrease to a number closer to zero, while the accuracy could also increase. After 25 epochs, the accuracy reached $98 \%$ and $97 \%$ respectively for the training and validation sets.

\section{E. Leaf disease classification}

Although the proposed deep neural network may achieve a high accuracy, it was created for the classification of small
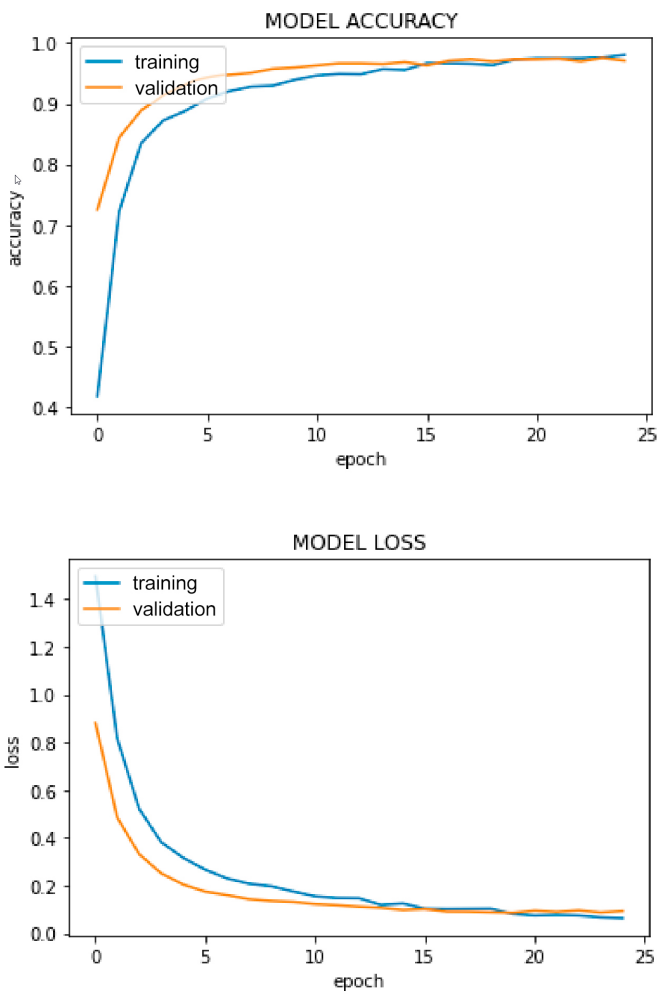

Fig. 4. Plots of accuracy and loss during training.

areas of each leaf, and not for the entire leaf. So, the final step of our proposed methodology is to combine the information obtained from each patch and indicate the soybean disease.

For the testing set, the patches were extracted not in the same way as for the training and validation sets. Patches of $224 \times 224$ were extracted in random locations, but to avoid the influence of the leaf structure (e.g. borders, veins, etc) and also to guarantee that most of the leaf area is well represented, the extraction respect the following rules:

- The minimum distance between the center of the all patches must be greater than 224 pixels, ensuring low or none overlap between them;

- The extracted patch must not contain a majority of background pixels, i.e. at least $50 \%$ of pixels should be within the leaf region.

- The number of extracted patches $N$ is given by:

$$
N=\left\lceil\frac{\text { Leaf Area } * 0.65}{224^{2}}\right\rceil,
$$

where \lceil\rceil denotes the ceiling function. Considering this equation, patches are extracted until the summation of their areas correspond to $65 \%$ of the leaf area.

An example of the patch extraction process can be seen in the Fig. 5.

Then, the class of each extracted patch is inferred using the retrained InceptionNetV3 and we compute the percentage of patches that were classified to each class. These percentages 


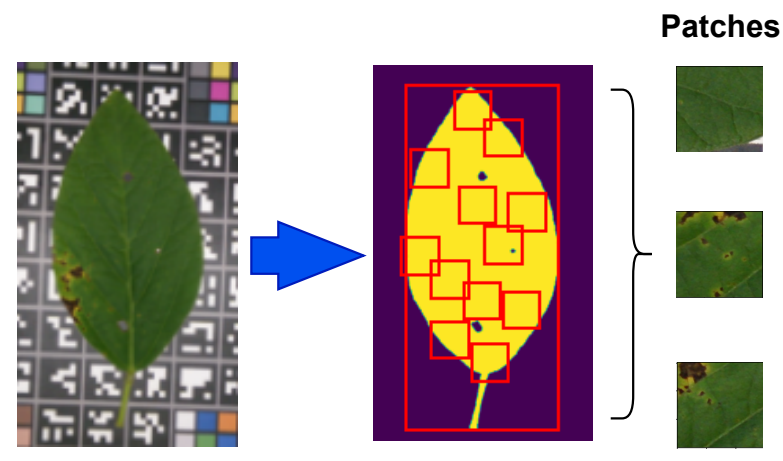

Fig. 5. Example of Patches Extraction.

create a vector with 6 numbers, representing the leaf. The vectors are then used as the input for a Support Vector Machine (SVM) classifier with linear kernel. Using a 5-fold crossvalidation for the testing dataset, the trained SVM were able to classify each leaf with accuracy of $98 \%$. An illustration of this whole process can be seen in Fig. 6.

\section{RESUlTS AND Discussion}

In Table IV, we present the obtained F-score, precision and recall for each of the 5 soybean leaf diseases using the proposed architecture. As can be seen, we were able to correct classify more than $90 \%$ of the images for all diseases.

TABLE IV

PRECISION, RECALL AND F-SCORE FOR EACH OF THE 5 DISEASES

\begin{tabular}{|l|c|c|c|}
\hline Class & Precision & Recall & F-score \\
\hline Soybean Mosaic Virus & 1.00 & 1.00 & 1.00 \\
\hline Bacterial Blight & 0.92 & 1.00 & 0.96 \\
\hline Rust & 1.00 & 1.00 & 1.00 \\
\hline Downy Mildew & 1.00 & 0.88 & 0.94 \\
\hline Powdery Mildew & 0.98 & 0.96 & 0.97 \\
\hline
\end{tabular}

Moreover, it is important to observe that SVM was used to improve the robustness of the whole model. If we apply just a majority voting, i.e. consider the disease with higher percentage from the extracted patches, the result would be different for only three images of the testing set, and it would also result more than $90 \%$ of correctly classified images for all diseases.

Barbedo et al. [8] obtained his dataset from the same source as ours, and although we cannot be sure, we believe that the images used in our experiments are the same as them for 4 leaf diseases. The comparison between our results and theirs for these 4 classes are presented in Table V. We believe that our approach achieves more precise results specially because of the feature extraction based on a convolutional neural network, which is able to better represent the characteristics of different level of degradations, while Barbedo et al. [8] used color histograms, which could make the classification step more difficult. And, another factor that was very important was the use of patches, increasing the number of samples for the training process and creating a more robust model.
TABLE V

COMPARISON OF OBTAINED PRECISIONS FOR BARBEDO ET AL. [8] AND OUR APPROACH

\begin{tabular}{|l|c|c|}
\hline Class & Barbedo et al. [8] & Ours \\
\hline Bacterial Blight & $24 \%$ & $\mathbf{9 2 \%}$ \\
\hline Rust & $94 \%$ & $\mathbf{1 0 0 \%}$ \\
\hline Downy Mildew & $60 \%$ & $\mathbf{1 0 0 \%}$ \\
\hline Powdery Mildew & $92 \%$ & $\mathbf{9 8 \%}$ \\
\hline
\end{tabular}

Regarding the other previous approaches for the classification of soybean leaf diseases, we present a comparison in Table VI. As can be seen, our method requires much lower number of images to achieved as precise or better results.

TABLE VI

COMPARISON WITH OTHER STATE-OF-THE-ART APPROACHES

\begin{tabular}{|l|c|c|}
\hline Autor & Avg. Leafs Per Class & Precision \\
\hline Pires et al. [4] & 300 & $98 \%$ \\
\hline Karlekar et al. [9] & 1077 & $97 \%$ \\
\hline Ours & $\mathbf{4 3}$ & $\mathbf{9 8 \%}$ \\
\hline
\end{tabular}

\section{Conclusion}

This paper proposed a robust and precise methodology for the classification of soybean leaf diseases. Using transfer learning from a renowned convolutional neural network, we trained a model able to classify patches from the original images and this classification was used in a Support Vector Machine to identify the disease.

The preliminary experimental results suggest that our approach can potentially be more robust than comparable methods available in the literature.

\section{ACKNOWLEDGMENT}

We would like to thank EMBRAPA for providing the image dataset.

\section{REFERENCES}

[1] EMBRAPA, "Soja em números (safra 2020/21)," tech. rep., Londrina, 2021.

[2] A. A. B. Junior, M. H. Hirakuri, J. C. Franchini, H. Debiasi, and R. H. Ribeir, "Análise da área, produção e produtividade da soja no brasil em duas décadas (1997-2016)," Londrina, 2017.

[3] J. F. J. Grigolli, "Manejo de doenças na cultura da soja," Tecnologia \& Produção Soja 2014/2015, 2015.

[4] R. D. L. Pires, D. N. Gonçalves, J. P. M. Oruê, W. E. S. Kanashiro, J. F. Rodrigues, B. B. Machado, and W. N. Gonçalves, "Local descriptors for soybean disease recognition," Computers and Electronics in Agriculture, vol. 125, pp. 48-55, 2016.

[5] A. dos Santos Ferreira, "Redes neuras convolucionais profundas na detecção de plantas daninhas em lavouras de soja," Master's thesis, Universidade Federal Do Mato Grosso Do Sul, 2017.

[6] A. Fuentes, S. Yoon, S. C. Kim, and D. S. Park, "A robust deep-learningbased detector for real-time tomato plant diseases and pests recognition," Sensors (Basel, Switzerland), vol. 17, 2017.

[7] S. M. Hassan, A. K. Maji, M. Jasiński, Z. Leonowicz, and E. Jasińska, "Identification of plant-leaf diseases using cnn and transfer-learning approach," Electronics, vol. 10, no. 12, 2021.

[8] J. G. A. Barbedo and C. V. Godoy, "Automatic classification of soybean diseases based on digital images of leaf symptoms," in $X$ congresso Brasileiro De Agroinformática, 2015.

[9] A. Karlekar and A. Seal, "Soynet: Soybean leaf diseases classification," Comput. Electron. Agric., vol. 172, p. 105342, 2020. 
Patches Classification

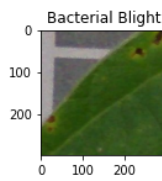

Bacterial Blight

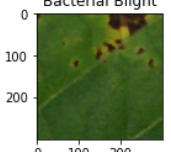

$100 \quad 200$

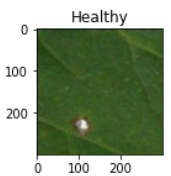

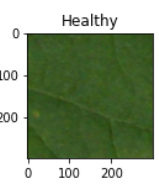

Bacterial Blight

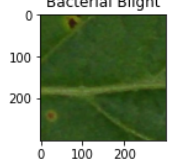

Bacterial Blight

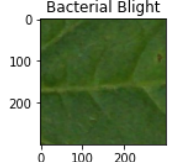

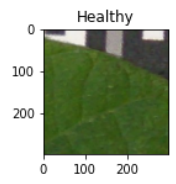

Soybean Mosaic Virus

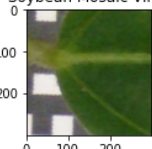

100200

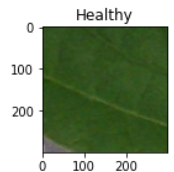

Fig. 6. Example of vectorization and its subsequent final classification.
Vector
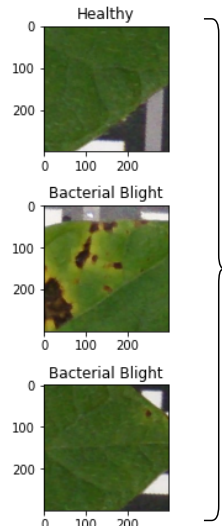

0.417

[10] C. Szegedy, V. Vanhoucke, S. Ioffe, J. Shlens, and Z. Wojna, "Rethinking the inception architecture for computer vision," 2015. 